\title{
Potentially inappropriate medications in relation to length of nursing home stay among older adults
}

\author{
Eva Sönnerstam ${ }^{1 *}$, Maria Gustafsson ${ }^{1}$ and Hugo Lövheim ${ }^{2}$
}

\begin{abstract}
Background: To investigate the use of potentially inappropriate medications and their relation to the length of nursing home stay among older adults.

Methods: Questionnaire surveys using the Multi-Dimensional Dementia Assessment scale were sent out to all nursing homes in Västerbotten county in northern Sweden in 2007 and 2013. In total, 3186 adults (1881 from 2007 and 1305 from 2013) $\geq 65$ years old were included and $71.8 \%$ of those had cognitive impairment. Potentially inappropriate medications were identified using drug-specific quality indicators according to Swedish National Board of Health and Welfare.

Results: Potentially inappropriate medications were used by $48.0 \%$ of the 2007 study sample and by $28.4 \%$ of the 2013 study sample. The prevalence of glibenclamide use 2007 and antipsychotic drug use 2013 increased linearly $\left(\beta=0.534 \mathrm{E}^{-3}, 95 \% \mathrm{Cl}: 0.040 \mathrm{E}^{-3}-0.103 \mathrm{E}^{-2}, p=0.034\right.$ and $\beta=0.155 \mathrm{E}^{-2}, 95 \% \mathrm{Cl}: 0.229 \mathrm{E}^{-3}-0.288 \mathrm{E}^{-2}, p=0.022$, respectively) with the length of nursing home stay. No significant association was found between the prevalence of propiomazine, codeine, long-acting benzodiazepines, anticholinergics, NSAIDs, tramadol or the total use of potentially inappropriate medications and the length of stay in nursing homes in 2007 or 2013 . Antipsychotics were the most commonly prescribed of the drug classes investigated and used by $22.6 \%$ of the residents 2007 and by $16.0 \%$ of the residents 2013.

Conclusions: These results indicate that treatment with potentially inappropriate medications is common among older adults living in nursing homes, but it seems to be related to the length of nursing home stay only to a smaller extent. Drug treatment should regularly be reviewed and followed-up among nursing home residents regardless of their length of nursing home stay, in order to prevent unnecessary adverse events.
\end{abstract}

Keywords: Older adults, Major neurocognitive disorders, Potentially inappropriate medications, Nursing homes, Length of stay

\section{Background}

Older adults living in nursing homes have more comorbidities compared to their counterparts in the wider community $[1]$ and major neurocognitive disorders are

*Correspondence: eva.sonnerstam@umu.se

${ }^{1}$ Department of Integrative Medical Biology, Umeå University, SE-901

87 Umeå, Sweden

Full list of author information is available at the end of the article common [2]. The number of older adults with major neurocognitive disorders increases exponentially after the age of 65 and it is a common cause of being admitted to a nursing home $[3,4]$. Consequently, as the number of older adults grow in society, so does the number of older adults with major neurocognitive disorders and those living in nursing homes [3]. 
Older adults in nursing homes and especially those with major neurocognitive disorders, are vulnerable to drugs and more prone to experience adverse drug reactions and hospital admissions $[1,3,5]$. One study found that $18.8 \%$ of acute hospital admissions were due to adverse drug reactions [6]. Drug related problems are common among nursing home residents [7] and it is found that these problems often are preventable [8]. Nevertheless, nursing home residents are often subject to polypharmacy and are prescribed more potentially inappropriate medications (PIMs) such as psychotropic drugs, compared to community-dwelling older adults [1, 9-11]. Antipsychotics and benzodiazepines are two type of psychotropic drug classes that are commonly prescribed, especially to older adults experiencing behavioural and psychological symptoms in dementia (BPSD) [12]. More specifically, antipsychotics are utilized to treat agitation, aggression, delusions and hallucinations [13], i.e. behavioural disturbances associated with major neurocognitive disorders that nursing home residents often experience [4]. Although antipsychotics are associated with severe side effects such as extrapyramidal symptoms, impaired cognition, cerebrovascular events and even increased risk of mortality [14-16], they are often used among residents without appropriate indications $[1,12]$. Moreover, benzodiazepines increase the risk of fall accidents and cognitive impairment [17]. Other PIMs that are often prescribed are anticholinergic drugs which contribute to cognitive impairment and delirium [18].

Trends indicate that PIM use has increased over time among nursing home residents [19]. On the other hand, a decrease of antipsychotic drug use in recent years was found among nursing homes residents living in Norway [20] and Sweden [21-24]. A previous study has found that having a higher number of pro re nata prescriptions, i.e. prescribed drugs used as needed, is associated with length of nursing home stay above 2.1 years [25]. Moreover, it was found that having a higher number of medications prescribed is associated with the length of stay in nursing homes [26]. These findings are supported by a Belgian study conducted among older adults in nursing homes [27]. However, the association between the prevalence of PIMs and length of nursing home stay among residents has not yet been determined.

Therefore, we aimed to investigate the prevalence of PIMs and the association between PIM use and the length of stay in nursing homes among older residents using Swedish explicit quality indicators.

\section{Methods \\ Data collection}

To determine the association between the prevalence of PIM use and the length of stay in nursing homes, data were collected from two cross-sectional surveys that were sent to all residents living in nursing homes in the county of Västerbotten in northern Sweden. The same questionnaire was sent twice, in May 2007 and 2013 respectively, and included an individual assessment of the residents' condition the preceding week. This should be filled in by the member of the staff who knew the resident best and was completed without direct involvement of the residents. Written instructions how the assessments should be carried out were included in the questionnaire and the staff were informed to contact the research team by telephone if questions arose. Moreover, a registered nurse was always instructed to complete data about the residents' currently prescribed medications and attach a copy of the current drug list before the questionnaire was sent back. Information about when the resident was admitted to the nursing homes was also noted in the questionnaire. Results from these surveys have previously been reported [23, 24, 28-31].

\section{Data assessments}

The Multi-Dimensional Dementia Assessment Scale (MDDAS) was used to assess the residents' health condition [32]. This assessment scale has good inter- and intra-rater reliability [32] and measures cognition, BPSD, hearing, speech, vision, motor functions and activities of daily living (ADL).

The MDDAS covers 14 different psychological symptoms related to mental health and 25 different behavioural symptoms. Each of the item is rated on a three-point scale dependent on how often the symptoms occurs, i.e. at least once a day, once a week or never during the preceding week. The scale therefore measures a one-week prevalence of BPSD occurrence among the residents if dichotomised between never and at least once a week.

The ADL score is based on the residents' ability to cope with dressing, eating, hygiene and bladder and bowel control. Every ADL category ranges between 1 and 5 except bowel control, which ranges between 0 and 4. Therefore, the sum of the ADL score varies from 4 to 24 where a higher score indicates a higher ADL independence.

A scale developed by Gottfries and Gottfries was utilised to measure the level of cognitive function among the residents $[29,33]$. The scale consists of 27 items and ranges between 0 and 27 points. A score less than 24 indicates cognitive impairment and correlates with $90 \%$ sensitivity and $91 \%$ specificity [32] to the cut-off of $24 / 30$ used in the Mini-Mental State Examination (MMSE) [34]. The score was further categorised into mild cognitive impairment (score 16-23), moderate cognitive 
impairment (score 8-15) and severe cognitive impairment (score 0-7).

\section{Data extraction}

Older adults with an identifiable year noted on the questionnaire, indicating when the person moved in to the nursing home, were qualified for inclusion in the study. If no valid month was noted, the individual got a random value of 1-12, or 1-6 if they moved in to the nursing home the same year as the questionnaire was distributed.

Moreover, a random value of 1-30 was imputed for those residents who lacked a specified day. Thereafter, the length of nursing home stay was estimated by calculating the time difference between when the questionnaire was sent out and when the resident moved in to the nursing home. Some individuals, however, got a value $<0$. Consequently, these individuals were excluded. Finally, the length of nursing home stay was truncated as complete months for an individual with a value $\geq 0$.

\section{Definition of potentially inappropriate medications}

Drug-specific quality indicators, listed by the Swedish National Board of Health and Welfare, were used to identify PIMs among the residents' drug lists [16]. The following drugs or drug classes, listed as inappropriate regardless of indication, were included in the analysis: long-acting benzodiazepines, anticholinergic drugs, tramadol, propiomazine, codeine and glibenclamide. Moreover, Non-Steroidal Anti-Inflammatory Drugs (NSAIDs) and antipsychotic drugs (except lithium) were included. According to Swedish indicators correct and current indication is important in order to classify NSAIDs and antipsychotic drugs as inappropriate. This information was however not available. Because of the many side effects associated with these drugs [14] they were classified as inappropriate medications regardless of indication in accordance with another study [24].

The research team identified and grouped the drugs according to World Health Organization (WHO) Anatomical Therapeutic Index (ATC) drug classification system. PIM use was defined as having at least one of the specified drugs prescribed regardless of treatment duration. Seven drug names were introduced on the Swedish market after 2007 and were therefore only identified among the residents in 2013. Consequently, missing values arose for these substances among respondents in 2007. These missing values were recoded as "did not use" $(n=1881)$ to enable correct prevalence calculations for all identified substances. Moreover, the attached drug list only provided information about the residents' ongoing drug treatment. Consequently, pro re nata drugs could not be included in the analysis.

\section{Study population}

There were 3578 and 3210 older adults living in geriatric care in Västerbotten county in 2007 and 2013, respectively and the response rate was $85.8 \%(n=3070)$ in 2007 and $70.5 \%(n=2262)$ in 2013 . Geriatric and psychogeriatric hospital wards were classified as geriatric care in 2007 but not in 2013. Respondents from these units were therefore excluded $(n=99)$ from the study population to include a homogenous sample from both years. Those younger than 65 years old or those for whom no age was registered $(n=278)$, older adults with missing values on the Gottfries scale $(n=569)$ and older adults without a complete medication list $(n=238)$ were excluded. Residents with a length of nursing home stay exceeding 5 years $(n=962)$ were also excluded to minimise a healthy survivor effect and to assure that no resident was included twice in the present study. Finally, respondents from $2007(n=1881)$ and $2013(n=1305)$ were included. Those with missing values (ADL, $n=52$ (2007), $n=71$ (2013) and sex, $n=4$ (2007), $n=6$ (2013)) were excluded from the regression analyses. An additional file, including a flowchart (Fig. A1), shows the inclusion process in more detail [see Additional file 1].

\section{Statistics}

Dichotomous variables are reported as frequencies. Variables with normal distribution are presented as mean values with standard deviation (SD) and variables with skewed distribution are reported as median with interquartile range (IQR). The prevalence of older adults using the different PIMs, PIM classes and total PIMs were plotted against the length of nursing home stay for the individual 2007 and 2013 study samples. To investigate the association between the use of PIMs in older adults and the length of nursing home stay 2007 and 2013, separate multilinear regression analyses were fitted to and conducted for each PIM or PIM class according to classification in the indicators. Finally, a multilinear regression analysis was conducted to investigate the association between individuals with at least one of the PIMs regardless of classification, i.e. total PIMs and the length of nursing home stay. However, as the PIM groups are not independent of the total PIM analysis, of which they are subsets, and also that all single significant associations are interpreted cautiously, it was decided that a multiple testing approach would not be necessary. The prevalence of older adults using at least one of the different PIMs, PIM classes and total PIMs was entered as the dependent variable into the model. Length of stay in months was entered as an independent variable into the model and the analyses were adjusted according to sex (entered as a dichotomous variable), age, level of cognitive function, 
Table 1 Basic characteristics of the study samples 2007 and 2013

\begin{tabular}{|c|c|c|}
\hline & 2007 & 2013 \\
\hline Total number of older adults, $\mathrm{n}$ & 1881 & 1305 \\
\hline Women $^{\mathrm{a}}, \mathrm{n}(\%)$ & $1272(67.6)$ & $886(67.9)$ \\
\hline Age, mean $\pm S D$ & $84.2 \pm 6.7$ & $84.9 \pm 6.9$ \\
\hline Severe cognitive impairment ${ }^{\mathrm{b}}, \mathrm{n}(\%)$ & $357(19.0)$ & $217(16.6)$ \\
\hline Moderate cognitive impairment ${ }^{c}, \mathrm{n}(\%)$ & $486(25.8)$ & $349(26.7)$ \\
\hline Mild cognitive impairment ${ }^{\mathrm{d}}, \mathrm{n}(\%)$ & $512(27.2)$ & $367(28.1)$ \\
\hline No cognitive impairment ${ }^{\mathrm{e}}, \mathrm{n}(\%)$ & $526(28.0)$ & $372(28.5)$ \\
\hline Gottfries'score, mean $\pm S D$ & $16.4 \pm 8.5$ & $16.8 \pm 8.1$ \\
\hline ADL score $(4-24)^{f}$, mean $\pm S D$ & $15.8 \pm 6.1$ & $16.1 \pm 6.0$ \\
\hline Number of medications \pm SD & $7.8 \pm 3.5$ & $7.7 \pm 3.6$ \\
\hline $\begin{array}{l}\text { Length of nursing home stay - number } \\
\text { of months, IQR (Range) }\end{array}$ & $6.0-33.0(0-60)$ & $7.0-32.0(0-60)$ \\
\hline
\end{tabular}

SD Standard deviation, $A D L$ Activities of daily living, IQR Interquartile range

a Missing: 2007, $n=4 ; 2013, n=6$

${ }^{\mathrm{b}}$ Gottfries' scale 0-7

'Gottfries' scale 8-15

d'Gottfries' scale 16-23

e Gottfries' scale 24-27

${ }^{f}$ Missing: 2007, $n=52 ; 2013, n=71$

ADL and number of medications (all entered as continuous variables). For comparison purpose, separate analyses were performed to investigate the relationship between PIMs, PIM classes and total PIMs respectively and the length of nursing home stay in the 2007 and 2013 populations without adjustment for number of medications. Consequently, these analyses were only adjusted for sex, age, level of cognitive function and ADL. Moreover, a supplementary multilinear regression analysis was conducted, investigating the association between number of medications and length of nursing home stay for the 2007 and 2013 populations. Number of medications was entered as a dependent variable and length of stay in months was entered as an independent variable into the model. These analyses were adjusted for sex, age, level of cognitive function and ADL. Only complete cases ( $n=1825$ (2007) and $n=1228$ (2013)) were included in the regression analyses. Linear regression curves were fitted to the data when the length of nursing home stay was statistically significant $(p<0.05)$. Moreover, the unstandardized $\beta$ for length of stay was presented together with 95\% confidence interval (CI) and the $p$-value. All statistical analyses were performed using IBM SPSS Statistics version 26 .

\section{Results}

Basic characteristics of the residents are listed in Table 1. Two thirds were women and the mean age was 84.2 years $( \pm 6.7)$ and 84.9 years $( \pm 6.9)$ in the 2007 and 2013 study samples, respectively. The length of nursing home stay ranged between 0 and 60 months for both study samples and $50 \%$ of the older adults had stayed for 6.0 33.0 months (IQR) in 2007 and 7.0-32.0 months (IQR) in 2013. Seven out of $10,72.0 \%$, of the 2007 study population and $71.5 \%$ of the 2013 study population had cognitive impairment according to Gottfries' score. The mean ADL score was $15.8( \pm 6.1)$ among the residents 2007 and 16.1 ( \pm 6.0 ) among the 2013 study population.

By using explicit quality indicators to identify PIM use among the residents, we found out that the prevalence of older adults using glibenclamide in 2007 and the prevalence of older adults using antipsychotic drugs in 2013 increased linearly $\left(\beta=0.534 \mathrm{E}^{-3}, 95 \% \mathrm{CI}\right.$ : $0.040 \mathrm{E}^{-3}-0.103 \mathrm{E}^{-2}, \quad p=0.034$ and $\beta=0.155 \mathrm{E}^{-2}, 95 \%$ CI: $0.229 \mathrm{E}^{-3}-0.288 \mathrm{E}^{-2}, p=0.022$, respectively) as the length of stay in nursing homes increased (Fig. 1.6a and $1.8 \mathrm{~b})$ when adjusted for age, sex, level of cognitive function, ADL and number of medications. However, no significant association was found between the prevalence of residents using long-acting benzodiazepines, anticholinergic drugs, tramadol, propiomazine, codeine, NSAIDs or the total use of PIMs and the length of stay in nursing homes for neither study population (Fig. 1.1-5, 1.7 and 1.9). Moreover, no significant association was found between the prevalence of residents using glibenclamide in 2013 or for the prevalence of residents using antipsychotic drugs in 2007 and the length of stay in nursing homes (Fig. 1.6b and 1.8a).

An additional file provides results for supplementary analyses (Fig. A2), presenting that the association between the prevalence of tramadol use, glibenclamide use and the use of at least one PIM and the length of nursing home stay were significant in $2007\left(\beta=0.797 \mathrm{E}^{-3}\right.$, 95\% CI: $0.121 \mathrm{E}^{-3}-0.147 \mathrm{E}^{-2}, \quad p=0.021 ; \beta=0.636 \mathrm{E}^{-3}$, 95\% CI: $0.143 \mathrm{E}^{-3}-0.113 \mathrm{E}^{-2}, p=0.011$ and $\beta=0.157 \mathrm{E}^{-2}$, $95 \%$ CI: $0.188 \mathrm{E}^{-3}-0.296 \mathrm{E}^{-2}, \quad p=0.026$, respectively), when number of medications was removed as an independent variable from the model. Moreover, the prevalence of older adults using antipsychotic drugs was still significantly associated with length of nursing home stay in $2013\left(\beta=0.162 \mathrm{E}^{-2}, 95 \% \mathrm{CI}: 0.281 \mathrm{E}^{-3}-0.295 \mathrm{E}^{-2}\right.$, $p=0.018$ ) when the analysis only was adjusted for age, sex, level of cognitive function and ADL. Additional analyses (Fig. A3) found a significant linear association between the number of medications and length of nursing home stay among participants in $2007(\beta=0.025,95 \%$ CI: $0.015-0.034, p<0.001$ ) [See Additional file 2].

Table 2 presents the number and frequencies of residents using PIMs. In total, $48.0 \%$ of the 2007 study population and $28.4 \%$ of the 2013 study population were prescribed at least one of the identified PIMs. The most commonly used drug class among the residents was 

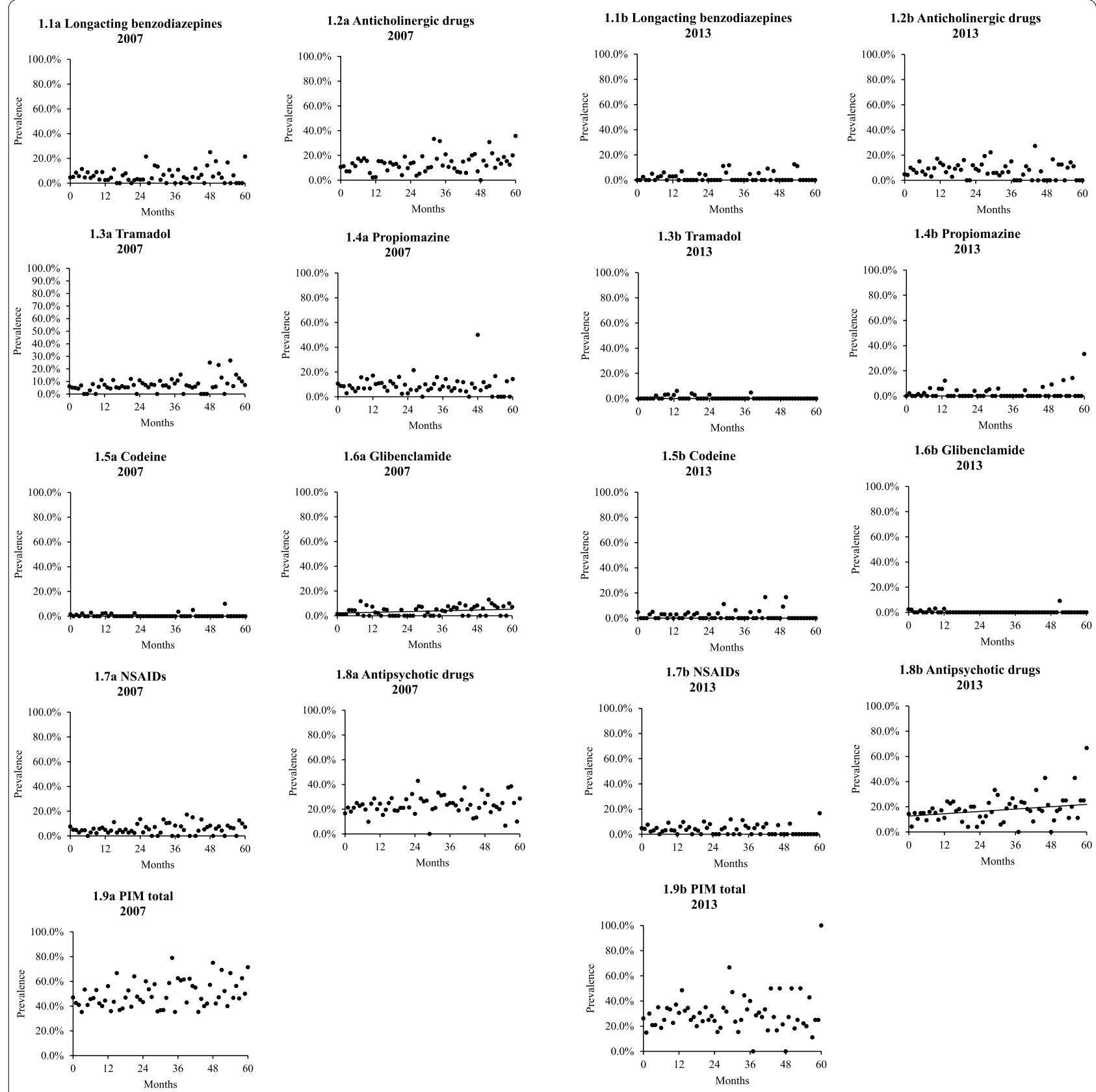

Fig. 1 The prevalence of older adults using PIMs in relation to length of stay in months. NSAIDs: Non-Steroidal Anti-Inflammatory Drugs; PIM: Potentially inappropriate medication

antipsychotics where $22.6 \%(n=426)$ and $16.0 \%(n=209)$ of the older adults in the 2007 and 2013 study samples, respectively, were affected. Risperidone $(n=178,9.5 \%$ (2007); $n=91,7.0 \%(2013))$, olanzapine $(n=92,4.9 \%$ (2007); $n=54,4.1 \%(2013))$ and haloperidol $(n=89,4.7 \%$ (2007); $n=25,1.9 \%$ (2013)) were the most commonly prescribed substances within that drug class. Anticholinergic drugs were the second most commonly prescribed drug class and were identified among $12.7 \%(n=238)$ of the residents in 2007 and among $8.0 \%(n=105)$ of the residents in 2013. Hydroxyzine $(n=56,3.0 \%$ (2007); $n=9,0.7 \%$ (2013)), ketobemidone and antispasmodics $(n=36,1.9 \%$ (2007); $\mathrm{n}=23,1.8 \%$ (2013)) and amitriptyline ( $n=32,1.7 \%$ (2007); $n=19,1.5 \%$ (2013)), were the most commonly used drugs. Furthermore, propiomazine 
Table 2 Frequency of residents using each of the identified PIM or PIM classes 2007 and 2013

\begin{tabular}{|c|c|c|}
\hline & 2007 & 2013 \\
\hline Total number of participants in study sample & 1881 & 1305 \\
\hline Long-acting benzodiazepines, $n(\%)^{\mathrm{a}}$ & $112(6.0)$ & $23(1.8)$ \\
\hline Diazepam (N05BA01), n (\%) & $15(0.8)$ & $6(0.5)$ \\
\hline Nitrazepam (N05CD02), n (\%) & $15(0.8)$ & $1(<0.1)$ \\
\hline Flunitrazepam (N05CD03), n (\%) & $85(4.5)$ & $18(1.4)$ \\
\hline Anticholinergic drugs, $\mathrm{n}(\%)^{\mathrm{a}}$ & $238(12.7)$ & $105(8.0)$ \\
\hline \multicolumn{3}{|l|}{ Gastrointestinal agents, anticholinergic } \\
\hline Glycopyrronium (A03AB02), n (\%) & 0 & $2(0.2)$ \\
\hline \multicolumn{3}{|l|}{ Anticholinergic antiemetics } \\
\hline Scopolamine (A04AD01), n (\%) & $3(0.2)$ & $3(0.2)$ \\
\hline \multicolumn{3}{|l|}{ Antiarrhythmics class $1 \mathrm{~A}$} \\
\hline Disopyramide (C01BA03), n (\%) & $2(0.1)$ & 0 \\
\hline \multicolumn{3}{|l|}{ Urinary antispasmodics (excl G04BD12) } \\
\hline Tolterodine (G04BD07), n (\%) & $24(1.3)$ & $4(0.3)$ \\
\hline Solifenacin (G04BD08), n (\%) & $1(<0.1)$ & $2(0.2)$ \\
\hline Darifenacin (G04BD10), n (\%) & $1(<0.1)$ & $1(<0.1)$ \\
\hline Fesoterodine (G04BD11), n (\%) & 0 & $2(0.2)$ \\
\hline \multicolumn{3}{|l|}{ Opiates and opioids in combination with antispasmodics } \\
\hline Ketobemidone and antispasmodics (N02AG02), n (\%) & $36(1.9)$ & $23(1.8)$ \\
\hline \multicolumn{3}{|l|}{ Anticholinergic anti-Parkinsonian drugs } \\
\hline Trihexyphenidyl (N04AA01), n (\%) & $5(0.3)$ & $4(0.3)$ \\
\hline Biperiden (N04AA02), $n(\%)$ & $10(0.5)$ & $2(0.2)$ \\
\hline \multicolumn{3}{|l|}{ Antipsychotic drugs } \\
\hline Levomepromazine (N05AA02), n (\%) & $21(1.1)$ & $13(1.0)$ \\
\hline Chlorprothixene (N05AF03), n (\%) & $1(<0.1)$ & 0 \\
\hline Clozapine (N05AH02), n (\%) & $13(0.7)$ & $3(0.2)$ \\
\hline \multicolumn{3}{|l|}{ Anxiolytics } \\
\hline Hydroxyzine (N05BB01), n (\%) & $56(3.0)$ & $9(0.7)$ \\
\hline \multicolumn{3}{|l|}{ Antidepressants, non-selective monoamine reuptake inhibitors } \\
\hline Clomipramine (N06AA04), n (\%) & $6(0.3)$ & $1(<0.1)$ \\
\hline Amitriptyline (N06AA09), $n$ (\%) & $32(1.7)$ & $19(1.5)$ \\
\hline \multicolumn{3}{|l|}{ Antihistamines } \\
\hline Clemastine (R06AA04), n (\%) & $14(0.7)$ & $10(0.8)$ \\
\hline Alimemazine (R06AD01), n (\%) & $29(1.5)$ & $11(0.8)$ \\
\hline Promethazine (R06AD02), n (\%) & $4(0.2)$ & $2(0.2)$ \\
\hline Meclozine (R06AE05), $n$ (\%) & 0 & $1(<0.1)$ \\
\hline Tramadol (N02AX02), n (\%) & $119(6.3)$ & $10(0.8)$ \\
\hline Propiomazine (N05CM06), n (\%) & $164(8.7)$ & 25 (1.9) \\
\hline Codeine, $n(\%)$ & $13(0.7)$ & $23(1.8)$ \\
\hline Paracetamol/codeine (N02AJ06), n (\%) & $13(0.7)$ & $21(1.6)$ \\
\hline Codeine (R05DA04), $n$ (\%) & 0 & $2(0.2)$ \\
\hline Glibenclamide (A10BB01), $n$ (\%) & $62(3.3)$ & $7(0.5)$ \\
\hline NSAIDs (COX-inhibitors), $n(\%)^{\mathrm{a}}$ & $97(5.2)$ & $48(3.7)$ \\
\hline Indometacin (M01AB01), n (\%) & $1(<0.1)$ & 0 \\
\hline Diclofenac (M01AB05), n (\%) & $25(1.3)$ & $16(1.2)$ \\
\hline Tenoxicam (M01AC02), n (\%) & $1(<0.1)$ & 0 \\
\hline Ibuprofen (M01AE01), n (\%) & $10(0.5)$ & $8(0.6)$ \\
\hline Naproxen (M01AE02), n (\%) & $26(1.4)$ & $10(0.8)$ \\
\hline Ketoprofen (M01AE03), n (\%) & $33(1.8)$ & $15(1.1)$ \\
\hline
\end{tabular}


Table 2 (continued)

\begin{tabular}{|c|c|c|}
\hline & 2007 & 2013 \\
\hline Celecoxib (M01AH01), $n$ (\%) & $1(<0.1)$ & 0 \\
\hline Etoricoxib (M01AH05), $n$ (\%) & $1(<0.1)$ & 0 \\
\hline Nabumetone (M01AX01), n (\%) & $4(0.2)$ & 0 \\
\hline Antipsychotic drugs, $n(\%)^{\mathrm{a}}$ & $426(22.6)$ & $209(16.0)$ \\
\hline Levomepromazine (N05AA02), n (\%) & $21(1.1)$ & $13(1.0)$ \\
\hline Dixyrazine (N05AB01), n (\%) & $2(0.1)$ & 0 \\
\hline Perphenazine (N05AB03), n (\%) & $6(0.3)$ & $2(0.2)$ \\
\hline Haloperidol (N05AD01), n (\%) & $89(4.7)$ & $25(1.9)$ \\
\hline Melperone (N05AD03), n (\%) & $14(0.7)$ & $10(0.8)$ \\
\hline Ziprasidone (N05AE04), n (\%) & $3(0.2)$ & $1(<0.1)$ \\
\hline Flupenthixol (N05AF01), n (\%) & $3(0.2)$ & $3(0.2)$ \\
\hline Chlorprothixene (N05AF03), n (\%) & $1(<0.1)$ & 0 \\
\hline Zuclopenthixol (N05AF05), n (\%) & $21(1.1)$ & $3(0.2)$ \\
\hline Clozapine (N05AH02), n (\%) & $13(0.7)$ & $3(0.2)$ \\
\hline Olanzapine (N05AH03), n (\%) & $92(4.9)$ & $54(4.1)$ \\
\hline Quetiapine (N05AH04), n (\%) & $7(0.4)$ & $18(1.4)$ \\
\hline Risperidone (N05AX08), n (\%) & $178(9.5)$ & $91(7.0)$ \\
\hline Aripiprazole (N05AX12), n (\%) & 0 & $1(<0.1)$ \\
\hline PIMs total, $n(\%)^{\text {b }}$ & $903(48.0)$ & $370(28.4)$ \\
\hline
\end{tabular}

NSAIDs Non-Steroidal Anti-Inflammatory Drugs, COX-inhibitors Cyclooxygenase inhibitors, PIMs Potentially inappropriate medications

${ }^{a}$ The frequency and prevalence differ from the sum of PIMs within the class, because some older adults used more than one PIM

${ }^{b}$ The frequency and prevalence differ from the sum of PIMs in total, because some older adults used more than one PIM from several PIM classes

was the third most commonly prescribed drug among them in 2007, $n=164$ (8.7\%). However, the third most commonly prescribed PIM class among the 2013 study population was NSAIDs, $n=48(3.7 \%)$. An additional file provides a supplementary table of all PIMs (Table A1) that were included from the Swedish quality indicators along with the frequency of residents using each PIM or PIM class [see Additional file 3].

\section{Discussion}

This paper describes how the prevalence of PIM use is associated to the length of stay in nursing homes. We did not find any association between total PIM use and increasing length of nursing home stay in none of the study populations and the prevalence of PIMs in the study population was already high among those newly admitted to the nursing homes both in 2007 and 2013. The prevalence of PIM use among the 2007 study population is similar to another where $44.3 \%$ of the population with mild or major neurocognitive disorders had at least one PIM prescribed before admission to nursing homes [35]. It is well known that those living in nursing homes and especially those with major neurocognitive disorders have more chronic conditions, more medications and PIMs, which support the findings of the high PIM prevalence in the present study and the significant association between number of medications and length of nursing home stay which was found among the 2007 study population $[1,9,10]$. Moreover, we found that total PIM use increased linearly with an increasing length of nursing home stay in the 2007 population when number of medications was removed from the model in the comparing analysis. Previous studies have found an increased risk of having PIMs when having a higher number of medications prescribed [36, 37]. PIM use is also found to be more common among frail residents than those with better health status at admission [35]. Moreover, the risk of being prescribed one or more PIMs after transition to a nursing home was found to be more common among frail older adults [35]. The result in the present study therefore implies that PIMs are also prescribed in the community or hospital settings before admission to nursing homes and that PIM use is, just to a lesser extent, associated with the length of nursing home stay and type of accommodation. This is supported by previous studies showing no association between PIM and type of accommodation among older adults admitted to hospital [36, 38, 39]. Consequently, the high PIM prevalence might be due to a higher disease burden and higher number of medications among those that have stayed longer and just, to a lesser extent, to the length of stay. This is supported by the supplementary analyses showing a significant 
linear association between the number of medications and increasing length of stay in nursing homes among the 2007 study population. Moreover, the associations between PIMs and length of nursing home that were found in the comparison analyses for the 2007 study sample, may be explained by the association between length of stay and number of medications. A high proportion, 72.0 and $71.5 \%$ of the residents in 2007 and 2013 respectively, have cognitive impairment, which is similar to a previous study [2]. PIMs in general are associated with increased risks of adverse drug reactions and consequently hospital admissions among older adults with cognitive impairment [6]. The high prevalence of PIMs among nursing home residents therefore warrants concern, regardless of their length of nursing home stay.

More than one fifth of the residents 2007 used some type of antipsychotic drug which is higher compared to one study [40] but lower compared to other studies [35, $41,42]$. In 2013, the prevalence of residents using antipsychotic drugs was lower, $16.0 \%$, and consequently lower compared to the previously mentioned studies [35, 4042]. No association was found between the prevalence of antipsychotic drug use and length of stay in nursing homes in 2007. This indicates that a significant proportion of the residents have antipsychotic drugs prescribed before admission to the nursing homes, in line with previous findings that antipsychotic drug use is high among those admitted from hospitals to nursing homes [40]. However, in 2013 a linear association was found between the prevalence of people using antipsychotic drugs and the length of stay in nursing homes. New prescribing habits and increasing prevalence of BPSD among those that have stayed longer in nursing homes might explain this relationship. BPSD is common among older adults with major neurocognitive disorders and consequently among residents living in nursing homes [4]. This can explain the high prevalence of antipsychotic drug use which contributes to the high prevalence of total PIM use in both study populations. Even if the antipsychotic drug use has decreased, this type of drug treatment is important to highlight because of its association with increased risk of mortality and cerebrovascular events among older adults with major neurocognitive disorders [14, 43].

Additionally, more than every tenth resident in 2007 and almost every tenth resident in 2013 were prescribed some type of anticholinergic drug in the present study. This contributed to the overall PIM prevalence among the residents and is worth highlighting due to the associated side effects such as impaired memory and delirium [18]. This type of drug treatment may therefore reinforce pre-existing symptoms among a group of adults where cognitive impairment is common and acetylcholine levels are lowered $[2,16]$.

Glibenclamide was the second substance for which there was a significant linear association with length of nursing home stay. This was found for the 2007 population. The small increase might be due to old prescribing habits, e.g. glibenclamide was more commonly prescribed historically and consequently this drug is more common among those who have stayed longer in nursing homes in 2007 if the drug has not been deprescribed. The utilized tool to define PIM use was revised in 2017 and was the first version that included glibenclamide as a PIM. The recommendations regarding glibenclamide use were therefore different in 2007 and 2013 when the surveys were distributed, which might explain the prevalence of glibenclamide use found in 2007.

Altogether, the changes observed in PIM prevalence in 2007 and 2013 might be due to the first revision of the Indicators for evaluating the quality of older people's drug therapy, developed by the Swedish National Board of Health and Welfare. This revision was made in 2010 [44]. Moreover, those counties which reduced the prevalence of PIMs included in the indicators by $10 \%$ among people 65 years or older, was offered a reward by the Swedish government in 2012. The decrease in prevalence might therefore be due to old prescribing habits, e.g. tramadol was more commonly prescribed 2007 compared to 2013. These trends are supported by a previous study [31]. Higher awareness of side effects and medication reviews may therefore explain the declining prescribing prevalence between the years. Nevertheless, it is important to continuously question the use of PIMs because of its associated side effects among the older adults. Adverse reactions become extra harmful among those with major neurocognitive disorders [16].

Strengths that should be mentioned are the large study populations of unselected nursing home populations, 2007 and 2013, and generally good quality of recorded data. Given the large sample size and robustness of linear regression, ordinary linear regression was assumed even if the distribution of length of nursing home stay was somewhat skewed. It should therefore be noted that the models probably are more affected by individuals with shorter length of stay. It should also be noted that no adjustment for multiple testing was made. Consequently, there is potential for increased false discovery rate. The response rate was $15 \%$ lower in 2013 than in 2007 which might be a reason for the smaller study sample in 2013. This may have contributed to the different PIM prevalence observed in 2007 and 2013. Moreover, explicit criteria were used when identifying PIMs in the present study and this is also 
one of the limitations, i.e. we did not have any information about diagnoses or indication of drug treatment. We also lacked information on how often the indication and drug treatment were evaluated and we did not have any information about the dose or duration of treatment. Even if this information was lacking, we chose to evaluate prevalence of antipsychotics and NSAIDs due to the serious side effects associated with this type of PIMs. Consequently, some treatment with antipsychotics and NSAIDs might have been appropriate. We also lacked information about pro re nata medications which may have affected the prevalence of long-acting benzodiazepines, tramadol and codeine that might be prescribed with this type of dosage. A healthy survivor effect might have influenced the prevalence of identified PIMs, i.e. those who have stayed longer in nursing home might be healthier than those who died before. In addition, due to having survived longer in nursing homes they may have been prescribed more medications and more PIMs.

\section{Conclusions}

Finally, we conclude that the length of stay just had a small impact on the prevalence of potentially inappropriate medications among nursing home residents. The high prevalence of potentially inappropriate medications found in the study suggests that future interventions should question this type of drug treatment among all residents regardless of their length of nursing home stay. Special focus should be directed to antipsychotic and anticholinergic drugs in order to prevent drug related side effects among nursing home residents.

\begin{abstract}
Abbreviations
ADL: Activities of daily living; ATC: Anatomical Therapeutic Index drug classification system; BPSD: Behavioural and psychological symptoms in dementia; $\mathrm{Cl}$ : Confidence interval; IBM: International Business Machines Corporation; IQR: Interquartile range; MDDAS: Multi-Dimensional Dementia Assessment Scale; MMSE: Mini-Mental State Examination; NSAIDs: Non-Steroidal AntiInflammatory Drugs; PIMs: Potentially inappropriate medications; SD: Standard deviation; SPSS: Statistical Package for Social Science; WHO: World Health Organization.
\end{abstract}

\section{Supplementary Information}

The online version contains supplementary material available at https://doi. org/10.1186/s12877-021-02639-3.

Additional file 1: Flowchart study participants, Flowchart showing the inclusion criteria for study participants 2007 and 2013.

Additional file 2: Supplementary analyses, Supplementary graphs presenting the relation between prevalence of PIM users and length of nursing home stay when number of medications was removed from the model. The relation between mean number of medications and length of nursing home stay is also presented.
Additional file 3: Supplementary PIM table, Supplementary table listing all PIMs that were included from the Swedish quality indicators and the frequency of residents using each PIM or PIM class.

\section{Acknowledgements}

Not applicable.

\section{Authors' contributions}

All authors were involved in the study concept and design. ES and HL were involved in and performed the statistical analysis. All authors contributed to the interpretation of the results. ES and MG were responsible for the initial draft. All authors critically revised, commented on the drafts, approved the final manuscript and take responsibility for the integrity of data and accuracy of the data analysis.

\section{Funding}

Open access funding provided by Umeå University. This study received financial support from the County Council of Västerbotten, Swedish Dementia Association and Swedish Brainpower. The funders had no role in the study design, data collection, analysis, interpretation of data, writing the manuscript, or decision to publish.

\section{Availability of data and materials}

Data can be obtained from the corresponding author on reasonable request.

\section{Declarations}

\section{Ethics approval and consent to participate}

The study was approved by the Regional Ethical Review Board in Umeå, Sweden (registration number 07-028 M [2007] and 2012-464-31 M [2013]) and performed in accordance with the ethical principles stated in the Declaration of Helsinki. An opt-out consent procedure was utilized in the present study. Written information was provided to all participants and posters with information about the study was available by the entrance of the nursing homes. Residents and their relatives could decline to participate if they did not want to participate in the study.

\section{Consent for publication}

Not applicable.

\section{Competing interests}

The authors declare that they have no competing interests.

\section{Author details}

${ }^{1}$ Department of Integrative Medical Biology, Umeå University, SE-901 87 Umeå, Sweden. ²Department of Community Medicine and Rehabilitation, Umeå University, 90187 Umeå, Sweden.

Received: 18 June 2021 Accepted: 16 November 2021

Published online: 22 January 2022

\section{References}

1. Fahey T, Montgomery AA, Barnes J, Protheroe J. Quality of care for elderly residents in nursing homes and elderly people living at home: controlled observational study. BMJ. 2003;326(7389):580.

2. Pasina L, Novella A, Cortesi L, Nobili A, Tettamanti M, lanes A. Drug prescriptions in nursing home residents: an Italian multicenter observational study. Eur J Clin Pharmacol. 2020;76(7):1011-9.

3. Ames D, O'Brien JT, Burns A. Dementia. 5th ed. New York: CRC Press Taylor \& Francis Group; 2017. p. 915.

4. Toot $S$, Swinson T, Devine M, Challis D, Orrell M. Causes of nursing home placement for older people with dementia: a systematic review and meta-analysis. Int Psychogeriatr. 2017;29(2):195-208.

5. Zeind CS. Carvalho, Michael G. applied therapeutics, the clinical use of drugs. 11th ed. China: Wolters Kluwer; 2018. p. 2374. 
6. Gustafsson M, Sjölander M, Pfister B, Jonsson J, Schneede J, Lövheim H. Drug-related hospital admissions among old people with dementia. Eur J Clin Pharmacol. 2016;72(9):1143-53.

7. Tjia J, Rothman MR, Kiely DK, Shaffer ML, Holmes HM, Sachs GA, et al. Daily medication use in nursing home residents with advanced dementia. J Am Geriatr Soc. 2010;58(5):880-8.

8. Gurwitz JH, Field TS, Judge J, Rochon P, Harrold LR, Cadoret C, et al. The incidence of adverse drug events in two large academic long-term care facilities. Am J Med. 2005;118(3):251-8.

9. Kristensen RU, Nørgaard A, Jensen-Dahm C, Gasse C, Wimberley T, Waldemar G. Polypharmacy and potentially inappropriate medication in people with dementia: a Nationwide study. J Alzheimers Dis. 2018;63(1):383-94.

10. Giron MS, Forsell Y, Bernsten C, Thorslund M, Winblad B, Fastbom J. Psychotropic drug use in elderly people with and without dementia. Int J Geriatr Psychiatry. 2001;16(9):900-6.

11. Jacquin-Piques A, Sacco G, Tavassoli N, Rouaud O, Bejot Y, Giroud M, et al. Psychotropic drug prescription in patients with dementia: nursing home residents versus patients living at home. J Alzheimers Dis. 2016;49(3):671-80.

12. Stevenson DG, Decker SL, Dwyer LL, Huskamp HA, Grabowski DC, Metzger ED, et al. Antipsychotic and benzodiazepine use among nursing home residents: findings from the 2004 National Nursing Home Survey. Am J Geriatr Psychiatry. 2010;18(12):1078-92.

13. Schneider LS, Dagerman K, Insel PS. Efficacy and adverse effects of atypical antipsychotics for dementia: meta-analysis of randomized, placebocontrolled trials. Am J Geriatr Psychiatry. 2006;14(3):191-210.

14. Gill SS, Bronskill SE, Normand SL, Anderson GM, Sykora K, Lam K, et al. Antipsychotic drug use and mortality in older adults with dementia. Ann Intern Med. 2007;146(11):775-86.

15. Ray WA, Chung CP, Murray KT, Hall K, Stein CM. Atypical antipsychotic drugs and the risk of sudden cardiac death. N Engl J Med. 2009:360(3):225-35.

16. The National Board of Health and Welfare. Indikatorer för god läkemedelsterapi hos äldre 2017 [Eng: Indicators for evaluating the quality of older people's drug therapy 2017]. Accessed 1 June 2018.

17. Picton JD, Marino AB, Nealy KL. Benzodiazepine use and cognitive decline in the elderly. Am J Health Syst Pharm. 2018;75(1):e6-e12.

18. Cancelli I, Beltrame M, Gigli GL, Valente M. Drugs with anticholinergic properties: cognitive and neuropsychiatric side-effects in elderly patients. Neurol Sci. 2009;30(2):87-92.

19. Morin L, Laroche ML, Texier G, Johnell K. Prevalence of Potentially Inappropriate Medication Use in Older Adults Living in Nursing Homes: A Systematic Review. J Am Med Dir Assoc. 2016:17(9):862.e1-9.

20. Selbæk G, Janus SIM, Bergh S, Engedal K, Ruths S, Helvik AS, et al. Change in psychotropic drug use in Norwegian nursing homes between 2004 and 2011. Int Psychogeriatr. 2018;30(3):385-94.

21. Lövheim H, Sandman PO, Kallin K, Karlsson S, Gustafson Y. Symptoms of mental health and psychotropic drug use among old people in geriatric care, changes between 1982 and 2000. Int J Geriatr Psychiatry. 2008;23(3):289-94.

22. Lövheim H, Sandman PO, Karlsson S, Gustafson Y. Changes between 1982 and 2000 in the prevalence of behavioral symptoms and psychotropic drug treatment among old people with cognitive impairment in geriatric care. Int Psychogeriatr. 2009;21(5):941-8.

23. Gustafsson M, Isaksson U, Karlsson S, Sandman PO, Lövheim H. Behavioral and psychological symptoms and psychotropic drugs among people with cognitive impairment in nursing homes in 2007 and 2013. Eur J Clin Pharmacol. 2016;72(8):987-94

24. Gustafsson M, Sandman PO, Karlsson S, Isaksson U, Schneede J, Sjölander $M$, et al. Reduction in the use of potentially inappropriate drugs among old people living in geriatric care units between 2007 and 2013. Eur J Clin Pharmacol. 2015;71(4):507-15.

25. Dörks M, Schmiemann G, Hoffmann F. Pro re nata (as needed) medication in nursing homes: the longer you stay, the more you get? Eur J Clin Pharmacol. 2016;72(8):995-1001.

26. Chen EY, Bell JS, Ilomaki J, Keen C, Corlis M, Hogan M, et al. Medication regimen complexity in 8 Australian residential aged care facilities: impact of age, length of stay, comorbidity, frailty, and dependence in activities of daily living. Clin Interv Aging. 2019;14:1783-95.

27. Ivanova I, Wauters M, Stichele RV, Christiaens T, De Wolf J, Dilles T, et al. Medication use in a cohort of newly admitted nursing home residents
(ageing@NH) in relation to evolving physical and mental health. Arch Gerontol Geriatr. 2018;75:202-8.

28. Lövheim H, Gustafson Y, Karlsson S, Sandman PO. Comparison of behavioral and psychological symptoms of dementia and psychotropic drug treatments among old people in geriatric care in 2000 and 2007. Int Psychogeriatr. 2011;23(10):1616-22.

29. Lövheim H, Gustafsson M, Isaksson U, Karlsson S, Sandman PO. Gottfries' cognitive scale for staff proxy rating of cognitive function among nursing home residents. J Alzheimers Dis. 2019:72(4):1251-60.

30. Gustafsson M, Sandman PO, Karlsson S, Gustafson Y, Lövheim H. Association between behavioral and psychological symptoms and psychotropic drug use among old people with cognitive impairment living in geriatric care settings. Int Psychogeriatr. 2013:25(9):1415-23.

31. Hemmingsson ES, Gustafsson M, Isaksson U, Karlsson S, Gustafson Y, Sandman $\mathrm{PO}$, et al. Prevalence of pain and pharmacological pain treatment among old people in nursing homes in 2007 and 2013. Eur J Clin Pharmacol. 2018;74(4):483-8.

32. Sandman PO, Adolfsson R, Norberg A, Nyström L, Winblad B. Longterm care of the elderly. A descriptive study of 3600 institutionalized patients in the county of Västerbotten, Sweden. Compr Gerontol A. 1988;2(3):120-32

33. Adolfsson R, Gottfries CG, Nyström L, Winblad B. Prevalence of dementia disorders in institutionalized Swedish old people. The work load imposed by caring for these patients. Acta Psychiatr Scand. 1981;63(3):225-44.

34. Folstein MF, Folstein SE, McHugh PR. "Mini-mental state". A practical method for grading the cognitive state of patients for the clinician. J Psychiatr Res. 1975;12(3):189-98.

35. Maclagan LC, Maxwell CJ, Gandhi S, Guan J, Bell CM, Hogan DB, et al. Frailty and potentially inappropriate medication use at nursing home transition. J Am Geriatr Soc. 2017;65(10):2205-12.

36. Sönnerstam E, Sjölander M, Gustafsson M. An evaluation of the prevalence of potentially inappropriate medications in older people with cognitive impairment living in northern Sweden using the EU(7)-PIM list. Eur J Clin Pharmacol. 2017;73(6):735-42.

37. Zuckerman IH, Hernandez JJ, Gruber-Baldini AL, Hebel JR, Stuart B, Zimmerman S, et al. Potentially inappropriate prescribing before and after nursing home admission among patients with and without dementia. Am J Geriatr Pharmacother. 2005:3(4):246-54.

38. Sönnerstam E, Sjölander M, Gustafsson M. Inappropriate prescription and renal function among older patients with cognitive impairment. Drugs Aging. 2016;33(12):889-99.

39. Sönnerstam E, Sjölander M, Lövheim H, Gustafsson M. Clinically relevant drug-drug interactions among elderly people with dementia. Eur J Clin Pharmacol. 2018;74(10):1351-60.

40. Rolland Y, Andrieu S, Crochard A, Goni S, Hein C, Vellas B. Psychotropic drug consumption at admission and discharge of nursing home residents. J Am Med Dir Assoc. 2012;13(4):407.e7-12.

41. Bronskill SE, Anderson GM, Sykora K, Wodchis WP, Gill S, Shulman Kl, et al. Neuroleptic drug therapy in older adults newly admitted to nursing homes: incidence, dose, and specialist contact. J Am Geriatr Soc. 2004;52(5):749-55.

42. Foebel A, Ballokova A, Wellens NI, Fialova D, Milisen K, Liperoti R, et al. A retrospective, longitudinal study of factors associated with new antipsychotic medication use among recently admitted long-term care residents. BMC Geriatr. 2015;15:128.

43. van lersel MB, Zuidema SU, Koopmans RT, Verhey FR, Olde Rikkert MG. Antipsychotics for behavioural and psychological problems in elderly people with dementia: a systematic review of adverse events. Drugs Aging. 2005;22(10):845-58.

44. The National Board of Health and Welfare. Indikatorer för god läkemedelsterapi hos äldre 2010. [Eng. Indicators for evaluating the quality of older people's drug therapy 2010].

\section{Publisher's Note}

Springer Nature remains neutral with regard to jurisdictional claims in published maps and institutional affiliations. 